\title{
ESCALAS E MÉTODOS DE ANÁLISE EM PESQUISA DE SATISFAÇÃO DE CLIENTES
}

\author{
Cláudia Maria de Oliveira Matsukuma ${ }^{1}$ \\ José Mauro da Costa Hernandez ${ }^{2}$
}

\begin{abstract}
Resumo: Este estudo compara a utilização das escalas de diferencial semântico, ordenação e soma constante e a análise de dados por meio de satisfação simples, análise de gap, modelo importânciasatisfação e abordagem multiplicativa na mensuração da satisfação com os atributos de um determinado produto ou serviço. $O$ objetivo do estudo é refletir sobre a utilização das escalas e técnicas, comparando os resultados obtidos em cada uma. Para testar as hipóteses formuladas, foram coletados dados utilizando-se um questionário estruturado hospedado em meio eletrônico e uma amostra obtida pelo sistema "bola-de-neve". Os resultados mostraram que as três escalas apresentam resultados significativamente diferentes apesar de as amostras serem parecidas. As quatro formas de análise também apresentam diferenças consideráveis com base nos mesmos dados. Conclui-se, portanto, que as escalas podem influenciar os resultados obtidos em uma coleta de dados, assim como as formas de análise apresentam resultados diferentes para os mesmos dados.
\end{abstract}

Palavras-chave: Satisfação do Consumidor. Serviço ao cliente. Pesquisa de Marketing.

\section{INTRODUÇÃO}

Os estudos de satisfação de clientes são cada vez mais comuns e sua importância já foi devidamente reconhecida pelas empresas. Ocorre, no entanto, que saber o quanto os clientes estão satisfeitos é interessante, mas não muito prático: se eles estão satisfeitos, o que está fazendo com que o estejam? Se há necessidade de cortar custos, como fazê-lo sem afetar - ou afetando o mínimo possível - a satisfação dos clientes? Se eles estão insatisfeitos, como reverter a situação?

Estudos de satisfação, portanto, dependem de avaliações individuais de determinados atributos da marca, produto ou serviço avaliado. Descobrir a satisfação em relação a cada atributo do objeto é parte importante do processo, mas não é todo o problema. Segundo Pilli (2004), na formulação teórica de estudos de satisfação e lealdade, a necessidade de identificação dos atributos com maior influência sobre a formação da atitude do consumidor no que diz respeito a marcas, produtos ou serviços é aceita pela quase totalidade dos profissionais e estudiosos do assunto. É por meio desses atributos que se pode entender a formação da satisfação do consumidor e tomar decisões estratégicas com maior segurança.

Isso significa que obter apenas a satisfação para cada um dos atributos não é suficiente. É necessário identificar a importância relativa de cada um dos atributos para a satisfação. Do ponto de vista gerencial, a implicação é clara: deve-se privilegiar os atributos mais importantes em detrimento dos menos importantes. Entretanto, se a necessidade de identificar a influência dos atributos sobre a satisfação geral é consenso entre os profissionais de pesquisa, o mesmo não se pode dizer sobre a melhor forma de se obter tal informação. Essa questão ainda não tem um consenso definido.

\footnotetext{
1 Escola de Comunicação e Artes, USP, São Paulo, SP.

2 Programa de Pós-Graduação em Administração, UNINOVE, São Paulo, SP. E-mail: jmhernandez@uninove.br
} 
Em princípio, a importância relativa pode ser obtida de duas formas: declarada e derivada. Para se obter a importância declarada, geralmente recorre-se a determinadas escalas como a de diferencial semântico, de ranking e de soma constante. Mas será que a escolha da escala pode influenciar os resultados sobre a importância relativa dos atributos?

De acordo com Pilli (2004), o problema da escolha de escalas está relacionado à forma como 0 entrevistado as interpretará. Ao analisar um objeto, o respondente processa mentalmente as informações disponíveis e suas respostas podem estar sujeitas a influências que comprometem a validade das medidas utilizadas.

A segunda questão que se coloca em relação às pesquisas de satisfação é o método de análise da satisfação para cada atributo. Para Fontenot et al. (2005), muitas são as formas de análise de dados que se propõe a isso, entre elas a medida de satisfação simples, a análise de gaps, o modelo I/S (Importância/Satisfação) e a abordagem multiplicativa. A dúvida aqui é se a escolha de uma forma particular de análise pode interferir na interpretação dos resultados da pesquisa.

Portanto, o primeiro objetivo deste trabalho é verificar se o tipo de escala com que se obtém a importância dos atributos - diferencial semântico, ordenamento e soma constante - influencia os resultados da ordem de importância dos atributos. O segundo objetivo é verificar se o método de análise da satisfação para cada atributo pode conduzir a conclusões diferentes sobre a satisfação dos indivíduos.

Para se atingir a esses objetivos, esta pesquisa foi estruturada em 5 seções: a primeira contém a revisão teórica sobre os diferentes tipos de escalas e de análises que vêm sendo empregadas em pesquisas de satisfação; a segunda descreve o método utilizado para testar as hipóteses formuladas na primeira seção; a terceira apresenta os resultados do teste empírico.; a quarta discute os resultados obtidos e a última sintetiza a conclusões obtidas no estudo.

\section{REVISÃO BIBLIOGRÁFICA}

\subsection{A PESQUISA DE SATISFAÇÃO DE CLIENTES}

Realizam-se pesquisas de satisfação de clientes porque existem evidências de que 0 aumento da satisfação gera lealdade, intenção de recompra e boa propaganda "boca-a-boca" (CHU, 2002). A satisfação é um índice que permite conhecer os clientes e, em conjunto com outras análises, indicar se o cliente é leal à empresa, se pretende migrar para o concorrente ou, ainda, se pretende abandonar 0 mercado. Ao que parece, o fator que gera lealdade e compromisso, por parte dos consumidores, é a entrega do produto e não o produto em si, pois os produtos inovadores são rapidamente replicados e está cada vez mais difícil diferenciá-los (CHAVDA, 2002). "O que é feito" parece importar menos do que "como é feito".

Assim, as pesquisas de satisfação, se bem utilizadas, podem auxiliar os profissionais de marketing a prever situações de risco e tomar decisões para evitá-las antes que aconteçam. Entretanto, o desenvolvimento das pesquisas de satisfação acabou gerando uma variedade enorme de maneiras de realizá-las, embora ainda não haja um consenso sobre qual a melhor forma. 


\subsection{PESQUISA DE SATISFAÇÃO DOM SERVIÇOS X PESQUISA DE SATISFAÇÃO COM PRODUTOS}

Existe uma diferenciação histórica entre pesquisa para avaliar produtos e a pesquisa para avaliar serviços. Os produtos podem ser avaliados mais objetivamente, uma vez que podem ser alterados e controlados de maneira mais "palpável". Além disso, há alguns anos, a indústria vivia o desafio de conseguir melhores níveis de qualidade na produção. Aparentemente, hoje, a qualidade tornou-se uma norma, minimizando a necessidade de se aprofundar estudos sobre os aspectos técnicos dos produtos. Uma vez testados e aprovados, estes são lançados e dificilmente geram problemas dessa ordem. Em contrapartida, a avaliação de serviços está mais sujeita a variações, já que estes são naturalmente intangíveis, dificultando a padronização e a redução de erros de forma consistente.

\subsection{PESQUISA DE SATISFAÇÃO UTILIZANDO ATRIBUTOS}

A diferenciação entre a pesquisa de satisfação com produtos e a pesquisa com serviços é especialmente importante quando considerados os atributos utilizados para avaliá-los. Ao passo que, de modo geral, um produto é avaliado em função de atributos físicos (cor, tamanho e forma, por exemplo), a avaliação dos serviços está mais sujeita à percepção individual. A percepção de "tempo de espera", por exemplo, pode variar de acordo com o serviço e a situação em que são utilizados.

A grande maioria das técnicas de pesquisa de satisfação (se não todas) utiliza algum tipo de avaliação de atributos. Isso ocorre porque as medidas-chave das pesquisas de satisfação - satisfação geral, recomendação, intenção de continuar usando ou comprando, intenção de migrar - podem indicar, até certo ponto, o nível de satisfação e lealdade dos consumidores. No entanto, tais medidas não se explicam por si só - "Por que os clientes estão ou não satisfeitos? Por que eles recomendam ou não 0 produto?" - elas simplesmente retratam a situação no momento em que a pesquisa está sendo realizada.

Em geral, os atributos são fornecidos pelo pesquisador, que os define com base em pesquisas qualitativas ou em dados primários. Entretanto, a definição dos atributos pelo pesquisador pode gerar um viés relacionado às informações fornecidas pelo instrumento de coleta. 0 consumidor costuma tomar decisões de compra com base em informações incompletas (KIVETZ; SIMONSON, 1997). Por exemplo, ao considerar a compra de um computador portátil, o consumidor pode tomar a decisão de compra baseando-se unicamente em atributos como velocidade, memória, peso e preço. No entanto, há outros atributos do equipamento que ele desconhece. Assim, quando o entrevistado, ele é estimulado a avaliar um produto com base em atributos fornecidos pelo pesquisador, sobre os quais pode não ter conhecimento ou conhecer apenas parcialmente. Dessa forma, é difícil de medir o impacto da falta de informação sobre o resultado de uma pesquisa.

De todo modo, as pesquisas de satisfação de clientes são baseadas em atributos, sejam estes definidos pelos pesquisadores ou pelos entrevistados. A questão a ser discutida é como esses atributos se relacionam com a satisfação geral dos clientes. 


\subsection{MÉTODOS PARA SE OBTER A IMPORTÂNCIA DOS ATRIBUTOS}

Muito já se discutiu e ainda se discute a respeito das melhores formas de obtenção da importância dos atributos em pesquisas de satisfação. Uma das principais questões é como se obter a importância relativa dos atributos. Basicamente, existem duas formas de se obter tal informação: derivando-se a importância relativa por algum método estatístico (importância derivada) ou a partir das declarações espontâneas do respondente (importância declarada). 0 foco deste estudo é a comparação de métodos alternativos para se obter a importância dos atributos por meio da declaração espontânea dos respondentes.

\subsubsection{Importância Derivada}

A importância derivada é obtida a partir da associação entre a avaliação individual de cada atributo e a avaliação geral. A importância dos atributos é então determinada estatisticamente por meio dessa relação. Hanson (1992) cita quatro maneiras de analisar a importância derivada: pelo coeficiente de correlação de Pearson, pelo coeficiente beta da regressão linear; pela multiplicação do coeficiente beta da regressão linear e o coeficiente de correlação de Pearson e pelo coeficiente de determinação parcial. Segundo Hanson (1992), o método mais utilizado é a análise de regressão da avaliação individual de todos os atributos tomados em conjunto sobre a avaliação geral do bem ou serviço. A importância relativa de cada atributo é obtida a partir do coeficiente de regressão de cada um dos atributos. Considerando que não é objeto deste estudo, o método de se obter a importância por meio da derivação não é abordado com profundidade no decorrer deste texto.

\subsubsection{Importância Declarada}

Ao contrário da importância derivada, na qual a importância dos atributos é obtida a partir de métodos estatísticos, a importância declarada solicita ao próprio entrevistado que avalie a importância dos atributos. A decisão de se utilizar a importância declarada deve ser tomada no início do planejamento da pesquisa, pois o instrumento de coleta deve ser desenhado de acordo com a maneira como os resultados serão avaliados. As escalas mais utilizadas para se obter a importância declarada são a escala de diferencial semântico, a escala de ordenamento e a escala de soma constante.

A escala de diferencial semântico é uma escala bipolar, ou seja, apenas os extremos da escala são descritos. Os entrevistados escolhem sua resposta sabendo que, quanto mais próximo de um dos extremos, mais o objeto avaliado se encontra próximo àquele extremo.

$\mathrm{Na}$ escala de ordenamento, o respondente avalia os atributos e os ordena de acordo com a importância relativa que ele dá a cada um. Por se tratar de uma escala comparativa, a principal vantagem dessa escala é forçar o entrevistado a priorizar alguns atributos em detrimento dos outros (MALHOTRA, 2001). No entanto, não é possível saber a "distância" entre os atributos na mente do consumidor.

$\mathrm{Na}$ escala de soma constante, o entrevistado distribui um número limitado de pontos entre os atributos, priorizando os mais importantes para a sua satisfação. Essa escala, além de fazer com que 0 entrevistado demonstre diferenciação entre os atributos, também permite saber qual é a distância entre eles. Suas principais desvantagens estão relacionadas à aplicação: 0 entrevistado precisa fazer a soma correta, atribuindo o número exato de pontos que foi determinado; o número de pontos não pode ser 
muito pequeno (pois pode causar problemas de arredondamento) nem muito grande (pois 0 entrevistado pode se confundir) (MALHOTRA, 2001, p. 245).

Durante a elaboração do instrumento de coleta, é preciso escolher qual escala será utilizada na pesquisa. A pergunta que um pesquisador se faria é se os resultados dependem da escala utilizada ou não. Sendo assim, a primeira hipótese deste estudo é a seguinte:

H1: As escalas de diferencial semântico, de ordenamento e de soma constante fornecem os mesmos resultados quando utilizadas para avaliação da importância de atributos.

\subsection{MÉTODOS DE ANÁLISE DA SATISFAÇÃO}

Para Fontenot et al. (2005), as principais formas de análise da satisfação com cada atributo são a satisfação simples, a análise de gaps, o Modelo I/S e a abordagem multiplicativa. A seguir, a descrição de cada uma dessas formas de análise.

\subsubsection{Satisfação simples}

Neste método, o entrevistado demonstra sua satisfação com os atributos, utilizando uma escala cujos extremos indicam se ele está muito satisfeito ou não está nada satisfeito com o item, ou seja, uma escala de diferencial semântico ancorada por dois extremos de satisfação (FONENOT et al., 2005). As notas médias dos itens são calculadas e aqueles com menores índices de satisfação devem ser priorizados. Esse método não leva em conta a importância dos atributos e não disponibiliza dados que ajudem a empresa a priorizar os atributos que geram maior impacto sobre a satisfação geral do cliente.

\subsubsection{Análise de Gap}

A análise de gap vai um pouco adiante ao levar em consideração a importância dos atributos para a análise (FONTENOT et al., 2005). A importância é obtida por meio de uma escala de diferencial semântico, na qual um extremo significa que 0 item é muito importante e o outro indica que é nada importante. Nesse método de análise, a importância é considerada como medida para indicar a expectativa de performance do cliente em relação a cada atributo.

\subsection{MODELO I/S (IMPORTÂNCIA/SATISFAÇÃO)}

O modelo I/S considera, simultaneamente, a satisfação com o atributo e a importância declarada pelos entrevistados. Para Fontenot et al. (2005), a diferença entre o modelo I/S e a análise de gap reside no fato de que "ao contrário da análise de gap, que examina a discrepância entre a importância e a satisfação, o modelo I/S analisa a relação entre os dois" (p. 42). O método consiste em plotar os dados de satisfação e importância declarada em um gráfico, com 4 quadrantes, para se identificar áreas de melhoria pela comparação entre o índice de satisfação e a importância dos atributos. 


\subsection{ABORDAGEM MULTIPLICATIVA}

A abordagem multiplicativa usa a importância como peso para os atributos e elimina a idéia de importância como indicador da expectativa de performance do cliente (FONTENOT et al., 2005). A diferença entre o índice mais alto possível de satisfação ("Totalmente satisfeito") e a percepção do consumidor sobre a performance do produto é utilizada para obter um índice de insatisfação, que é multiplicado pelo índice de importância. Assim como na análise de gap, em caso de empate, é preciso avaliar os atributos de acordo com seu índice de importância.

As quatro formas apresentadas podem ser utilizadas para analisar os dados de uma pesquisa que avalia a importância e a satisfação com os atributos. Fontenot et al. (2005) utilizam essas formas de análise para determinar quais são os atributos que requerem maior atenção das empresas.

No entanto, resta a pergunta: será que as quatro formas de análise fornecem os mesmos resultados? Dessa forma, a segunda hipótese deste estudo é a seguinte:

H2: A utilização dos métodos de análise satisfação simples, análise de gap, modelo I/S e abordagem multiplicativa indicam os mesmos atributos a serem priorizados.

\section{MÉTODO}

Neste capítulo, são discutidos os procedimentos utilizados para testar as hipóteses formuladas na seção anterior. São abordadas a composição da amostra, as etapas da elaboração dos questionários e as escalas utilizadas.

\subsection{PROCEDIMENTOS PARA A COLETA DOS DADOS}

O objeto escolhido para ser avaliado foi o website de relacionamentos Orkut (www.orkut.com). Nesse website, as pessoas se cadastram e passam a se relacionar virtualmente por meio das ferramentas disponíveis. Para se cadastrar, é necessário ter sido convidado. 0 Orkut foi escolhido como objeto pela conveniência de se coletar os dados e por se tratar de um serviço, o que, em princípio, torna a avaliação dos atributos uma tarefa mais complexa e adequada ao propósito deste estudo. Além disso, uma vez que é comum utilizar-se a técnica de bola de neve para divulgar informações via internet, solicitar que as pessoas colaborassem com a pesquisa seria relativamente natural para a comunidade do referido website.

Considerando que o objeto de análise era um website, pareceu natural que os dados fossem coletados por meio de um questionário eletrônico. Os questionários (ver detalhes na próxima seção) foram disponibilizados no endereço www.beezoo.net/pesquisa e ficaram disponíveis aos respondentes por 8 dias.

Para facilitar a divulgação da pesquisa, todos os entrevistados foram direcionados para um único endereço. Na pagina inicial havia uma introdução à pesquisa e algumas perguntas-filtro. Pessoas com menos de 14 anos; que não utilizavam o Orkut, utilizavam uma vez por mês ou menos foram excluídas da amostra.

Como estímulo, foram sorteados dois vales-compra - no valor de $\mathrm{R} \$ 50,00$ cada - entre os respondentes que completaram todo o questionário. 


\subsection{AMOSTRA}

Para a realização do estudo foi utilizado o processo de seleção de amostra do tipo "bola-deneve": um grupo de usuários do site Orkut recebeu um convite, por e-mail, para participar da pesquisa e repassá-lo aos seus amigos. Ao final da coleta de dados, 562 pessoas haviam se cadastrado na página inicial do site. A amostra final foi composta por 351 casos, o que resulta numa taxa de aproveitamento de $62 \%$. A maioria das perdas se deu por desistência durante o preenchimento do questionário (56\%) e pela eliminação no filtro (44\%).

A maior parte da amostra é composta por jovens entre 23 e 29 anos (56\%), os quais utilizam freqüentemente a Internet (65\% por mais de $1 \mathrm{~h} 30$ por dia; $59 \%$ da amostra acessam o Orkut diariamente.

\subsection{QUESTIONÁRIOS E ESCALAS}

Para se elaborar os questionários, o primeiro passo foi a escolha do conjunto de atributos pelos quais o website pudesse ser avaliado. Após a análise da literatura sobre a qualidade de Websites (por exemplo, YOO; DONTHU, 2001), uma profunda análise das características do Orkut e algumas conversas informais com membros dessa comunidade virtual, foram definidos 12 atributos. 0 processo de escolha dos atributos certamente é reflexo das idiossincrasias dos pesquisadores e muito semelhante ao método freqüentemente utilizado por pesquisadores profissionais de marketing. Embora tal método de seleção de atributos possa ser questionado do ponto de vista científico, o objetivo era colocar à prova um sistema frequentemente utilizado por empresas de pesquisa. Como o objetivo não era testar a validade dos atributos, mas o resultado proporcionado pelas escalas e pelos métodos de análise, considerou-se que essa forma seria válida.

Os doze atributos assim escolhidos estão descritos na Tabela 1, assim como os nomes das variáveis pelos quais estes atributos serão tratados no restante deste estudo.

\section{Tabela 1 - Atributos avaliados}

\begin{tabular}{ll|l}
\hline \multicolumn{2}{c|}{ ATRIBUTO } & NOME DA VARIÁVEL \\
\hline 1. & Instruções para a utilização do site & Instruções \\
\hline 2. & Conteúdo do FAQ (respostas para as perguntas mais comuns) & FAQ \\
\hline 3. & Disponibilidade de uma ferramenta para encontrar e manter contato com amigos usuários & Manter contato \\
\hline 4. & Possibilidade de participar de comunidades com pessoas que têm os mesmos interesses & Participar de comunidades \\
\hline 5. & Possibilidade de conhecer melhor as pessoas por meio do perfil cadastrado & Conhecer pessoas pelo perfil \\
\hline 6. & Possibilidade de cadastrar um perfil com informações variadas & Cadastrar perfil \\
\hline 7. & Possibilidade de classificar amigos como fãs e avaliar o quanto são legais, confiáveis e sensuais & Classificar amigos \\
\hline 8. & Possibilidade de saber se estou "conectado" a outras pessoas por meio das redes de amigos & Saber se estou conectado \\
\hline 9. & Visual (cores, tamanho e tipo de fonte, disposição dos elementos na tela) & Visual \\
\hline 10. & Facilidade de navegar pelo site de maneira eficiente e intuitiva, sem ficar "perdido" & Navegabilidade \\
\hline 11. & O site estar livre de erros em geral & Erros \\
\hline 12. & Segurança no cadastro de informações do perfil e das comunidades & Segurança \\
\hline
\end{tabular}


Para que as hipóteses fossem testadas, foram elaborados quatro questionários, sendo que cada um deles operacionalizava a medida de satisfação com o Orkut por meio de uma determinada escala.

A primeira parte dos questionários apresentava as perguntas-filtro (freqüência de uso do Orkut e idade), além da pergunta sobre freqüência de utilização da Internet. A segunda continha 7 perguntas: a primeira era uma medida de satisfação geral com o Orkut em que se utilizou uma escala de diferencial semântico de 10 pontos ( 1 = totalmente insatisfeito; 10 = totalmente satisfeito); a segunda pedia ao respondente justificasse a resposta anterior e as outras 4 diziam respeito às intenções de uso do Orkut no futuro (intenção de continuar usando o orkut, intenção de utilizar outro website de relacionamento, se recomendaria o Orkut para amigos e parentes; se costuma falar bem do Orkut). A sétima pergunta foi a única formulada de forma diferente em cada um dos quatro questionários.

$\checkmark$ Questionário 1 - Satisfação Simples - os respondentes foram orientados a avaliar a sua satisfação com cada um dos doze atributos da Tabela 1, utilizando uma escala de diferencial semântico de 10 pontos ( 1 = totalmente insatisfeito; 10 = totalmente satisfeito).

$\checkmark$ Questionário 2 - Escala de Diferencial Semântico - os respondentes foram convidados a avaliar a importância de cada um dos doze atributos para a sua satisfação com o website, utilizando uma escala de diferencial semântico de dez pontos (1 = nada importante; $10=$ muito importante).

$\checkmark$ Questionário 3 - Escala de Soma Constante - solicitou-se aos respondentes que atribuíssem 100 pontos aos doze atributos de acordo com a importância de cada um deles para sua satisfação.

$\checkmark$ Questionário 4 - Escala de Ordenamento - os respondentes foram orientados a ordenar os doze atributos de acordo com a importância que atribuíam a cada um deles para a sua satisfação com o website.

A distribuição final da amostra entre os 4 tipos de questionário foi a seguinte: 91 respostas para o questionário 1, 88 respostas para o questionário 2, 85 respostas para o questionário 3 e 85 respostas para o questionário 4.

Para verificar se havia diferença entre as amostras de cada um dos questionários, foram comparadas, por meio de testes qui-quadrado, a idade dos respondentes, a freqüência de uso da internet e a freqüência de uso do site Orkut. Esses testes demonstraram que as amostras não são significativamente diferentes.

\section{RESULTADOS}

Nessa seção são descritos os resultados obtidos com as análises das três escalas com que a importância foi mensurada e das quatro formas de análise da satisfação.

\subsection{SATISFAÇÃO GLOBAL E INTENÇÃO}

Os questionários tinham perguntas em comum que indicam a satisfação geral e medidas relacionadas à lealdade dos usuários. As respostas a essas perguntas demonstram que $59 \%$ dos entrevistados estão satisfeitos ou muito satisfeitos com o website e apenas $11 \%$ estão insatisfeitos ou 
muito insatisfeitos; a grande maioria (96\%) tem a intenção de continuar usando o Orkut, $81 \%$ não têm intenção de usar outro site, 93\% recomendaria o Orkut para seus amigos e parentes e mais de $86 \%$ costuma falar bem do Orkut. Esses resultados indicam um alto grau de satisfação com o Orkut.

\subsection{AVALIAÇÃO DA IMPORTÂNCIA RELATIVA DOS ATRIBUTOS}

Inicialmente, foram calculadas as médias de importância para cada atributo, segundo as diferentes escalas (diferencial semântico, soma constante e ordenamento), conforme descritos na Tabela 1. A seguir, as médias de importância para todos os atributos foram comparadas em pares para identificar diferenças estatísticas entre elas (anexos I, II e III). As conclusões que podem ser tiradas a partir destas quatro tabelas são as seguintes:

$\checkmark \mathrm{O}$ atributo mais importante segundo as três escalas é "manter contato". Na escala de diferencial semântico, esse atributo é tão importante quanto "segurança".

$\checkmark$ O segundo bloco de atributos em ordem de importância é composto por "segurança", "navegabilidade", "participar de comunidades", "conhecer pessoas pelo perfil" e "cadastrar perfil". A importância relativa de cada um destes atributos varia de escala para escala.

$\checkmark$ O terceiro bloco de atributos em ordem de importância é composto por "visual", "saber se estou conectado" e "instruções". Nas escalas de soma constante e ordenamento, o atributo "Erros" aparece nesse grupo, ao passo que, na escala de diferencial semântico 0 atributo "erros" está no bloco anterior.

$\checkmark$ Os atributos menos importantes segundo as três escalas são "FAQ" e "classificar amigos"

De modo geral, pode-se concluir que os atributos mais importantes e os menos importantes são adequadamente identificados segundo as três escalas. Entretanto, o nível de valorização dos atributos de importância intermediária varia de escala para escala. Assim, um pesquisador hipotético teria obtido resultados diferentes, dependendo da escala utilizada. Dessa forma, rejeita-se H1, isto é, a importância dos atributos varia de acordo com o tipo de escala utilizada.

Tabela 2 - Médias das Importâncias dos Atributos para a Satisfação com o Website

\begin{tabular}{l|c|c|c}
\hline & $\begin{array}{c}\text { ESCALA DE DIFERENCIAL } \\
\text { SEMÂNTICO }\end{array}$ & $\begin{array}{c}\text { ESCALA DE SOMA } \\
\text { CONSTANTE }\end{array}$ & $\begin{array}{c}\text { ESCALA DE } \\
\text { ORDENAMENTO }\end{array}$ \\
\hline Manter contato & 8,58 & 20,93 & 2,69 \\
\hline Segurança & 8,43 & 8,54 & 4,44 \\
\hline Erros & 8,07 & 6,68 & 7,05 \\
\hline Navegabilidade & 8,00 & 8,16 & 5,10 \\
\hline Participar de comunidades & 7,93 & 10,94 & 5,41 \\
\hline Conhecer pessoas pelo perfil & 7,33 & 8,69 & 6,43 \\
Cadastrar perfil & 6,76 & 8,13 & 6,23 \\
\hline Visual & 6,49 & 5,88 & 8,32 \\
\hline Saber se estou conectado & 6,45 & 7,00 & 6,85 \\
\hline Instruções & 6,22 & 5,53 & 7,69 \\
\hline FAQ & 5,36 & 5,02 & 8,54 \\
Classificar amigos & 4,92 & 4,48 & 9,25 \\
\hline Base & 88 & 85 & 85 \\
\hline
\end{tabular}




\subsection{AVALIAÇÃO DA SATISFAÇÃO COM OS ATRIBUTOS - DIFERENTES MÉTODOS DE ANÁLISE}

Nesta seção são analisados os resultados obtidos com os quatro métodos de análise da satisfação com os atributos.

\subsection{SATISFAÇÃO SIMPLES}

A técnica de Satisfação Simples, descrita por Fontenot et al. (2005), consiste apenas em calcular a satisfação média obtida para cada um dos atributos. Esses dados foram obtidos por intermédio do Questionário 1. A média de satisfação para cada um dos doze atributos encontra-se na Tabela 2.

Tabela 3 - Satisfação Média com Cada Atributo do Orkut

\begin{tabular}{l|c}
\hline \multicolumn{1}{c|}{ ATRIBUTO } & SATISFAÇÃO MÉDIA \\
\hline Participar de comunidades & 8,41 \\
\hline Cadastrar perfil & 7,37 \\
\hline Visual & 7,37 \\
\hline Contato & 7,33 \\
\hline Navegabilidade & 7,25 \\
\hline Conhecer pessoas pelo perfil & 7,11 \\
\hline Saber se estou conectado & 7,10 \\
\hline Classificar amigos & 6,74 \\
\hline Instruções & 6,42 \\
\hline FAQ & 6,32 \\
\hline Segurança & 5,04 \\
\hline Erros & 3,89 \\
\hline
\end{tabular}

Base: 91 respondentes do Questionário 1

Fontenot et al. (2005) sugerem que os atributos a serem privilegiados pela administração de marketing são aqueles com os quais os respondentes revelam menor satisfação. Nesse caso, em ordem de prioridade, a administração de marketing deveria cuidar dos "erros", "da segurança" e da "FAQ" do Orkut.

\subsection{ANÁLISE DE GAP}

A análise de gap consiste em calcular a diferença entre as médias de importância de cada atributo e de satisfação em relação aos mesmos. A satisfação simples foi obtida por intermédio do questionário 1 e a importância foi obtida através do questionário 2 (Tabela 3), conforme descrito anteriormente. De acordo com os resultados da análise de gap, o atributo com maior gap é "erros" seguido por "segurança" e "manter contatos". Quando o resultado da análise de gap é negativo significa que a satisfação com o atributo é maior do que a importância atribuída ao mesmo, indicando que não deve ser dada prioridade imediata ao atributo pela administração de marketing. 
Tabela 4 - Análise de Gap para os Atributos do Orkut

\begin{tabular}{l|c|c|c}
\hline & SATISFAÇÃO & IMPORTÂNCIA & GAPS \\
\hline Erros & 3,9 & 8,1 & 4,2 \\
\hline Segurança & 5,0 & 8,4 & 3,4 \\
\hline Manter contato & 7,3 & 8,6 & 1,2 \\
\hline Navegabilidade & 7,3 & 8,0 & 0,7 \\
\hline Conhecer pessoas pelo perfil & 7,1 & 7,3 & 0,2 \\
\hline Instruções & 6,4 & 6,2 & $-0,2$ \\
\hline Participar de comunidades & 8,4 & 7,9 & $-0,5$ \\
\hline Cadastrar perfil & 7,4 & 6,8 & $-0,6$ \\
\hline Saber se estou conectado & 7,1 & 6,5 & $-0,6$ \\
\hline Visual & 7,4 & 6,5 & $-0,9$ \\
\hline FAQ & 6,3 & 5,4 & $-1,0$ \\
\hline Classificar amigos & 6,7 & 4,9 & $-1,8$ \\
\hline
\end{tabular}

Base: 88 respondentes dos Questionário 1 e 2

\subsection{MODELO I/S (IMPORTÂNCIA/SATISFAÇÃO)}

O modelo I/S consiste em se plotar as médias de importância no eixo das abscissas de um gráfico e as médias da satisfação no eixo das ordenadas. 0 gráfico assim formado (Figura 1) pode ser dividido em quatro quadrantes.

No quadrante superior direito, a satisfação e a importância estão acima da média, indicando que não existe nenhum problema com o atributo e ele deve ser mantido como está.

No quadrante inferior direito, a importância está acima e a satisfação abaixo da média. Os atributos posicionados nesse quadrante precisam ser melhorados e devem ser considerados como prioridades pela administração de marketing.

No quadrante superior esquerdo, a importância está abaixo e a satisfação acima da média. Os atributos posicionados nesse quadrante estão com excedente.

Finalmente, no quadrante inferior esquerdo, a satisfação e a importância estão abaixo da média. Deve-se dar atenção aos atributos localizados neste quadrante, pois eventualmente eles podem ser tornar mais importantes.

Com base nos dados obtidos pelos questionários 1 e 2, o gráfico sugerido pelo modelo I/S foi construído e o resultado está descrito na Figura 1. De acordo com a análise sugerida acima, o único atributo que deve ser melhorado é o atributo "erros". O atributo "segurança" está no limite entre os quadrantes Manter e Melhorar.

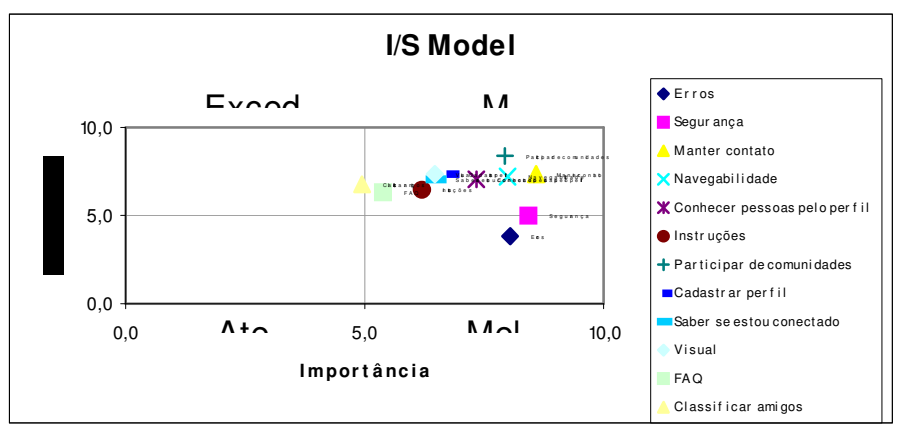

Figura 1 - Modelo I/S (Importância/Satisfação) para os Atributos do Orkut 


\subsection{ABORDAGEM MULTIPLICATIVA}

A abordagem multiplicativa pressupõe obter um índice de insatisfação para cada atributo e multiplicá-lo pela importância relativa. $O$ índice de insatisfação é obtido pelo cálculo da diferença entre a maior satisfação possível e a satisfação mensurada. Os resultados da abordagem multiplicativa para os atributos do Orkut estão descritos na Tabela 4.

Segundo esta análise, quanto maior o índice de insatisfação ponderado, maior a atenção que deve ser dada ao atributo. Os resultados demonstrados na Tabela 4 sugerem que 0 atributo com maior índice de insatisfação ponderado é "erros", seguido por "segurança", "manter contato".

Tabela 4 - Abordagem Multiplicativa para os Atributos do Orkut

\begin{tabular}{l|c|c|c|c|c}
\hline & $\begin{array}{c}\text { MAIOR } \\
\text { ÍNDICE } \\
\text { POSSÍVEL }\end{array}$ & SATISFAÇÃO & $\begin{array}{c}\text { ÍNDICE DE } \\
\text { INSATISFAÇÃO }\end{array}$ & IMPORTÂNCIA & $\begin{array}{c}\text { INSATISFAÇÃO } \\
\text { INONDERADA }\end{array}$ \\
\hline Erros & 10,0 & 3,9 & 6,1 & 8,1 & 49,41 \\
\hline Segurança & 10,0 & 5,0 & 5,0 & 8,4 & 42,00 \\
\hline Manter contato & 10,0 & 7,3 & 2,7 & 8,6 & 23,22 \\
\hline Instruções & 10,0 & 6,4 & 3,6 & 6,2 & 22,32 \\
\hline Navegabilidade & 10,0 & 7,3 & 2,7 & 8,0 & 21,60 \\
\hline Conhecer pessoas pelo perfil & 10,0 & 7,1 & 2,9 & 7,3 & 21,17 \\
\hline FAQ & 10,0 & 6,3 & 3,7 & 5,4 & 19,98 \\
\hline Saber se estou conectado & 10,0 & 7,1 & 2,9 & 6,5 & 18,85 \\
\hline Cadastrar perfil & 10,0 & 7,4 & 2,6 & 6,8 & 17,68 \\
\hline Visual & 10,0 & 7,4 & 2,6 & 6,5 & 16,90 \\
\hline Classificar amigos & 10,0 & 6,7 & 3,3 & 4,9 & 16,17 \\
\hline Participar de comunidades & 10,0 & 8,4 & 1,6 & 7,9 & 12,64 \\
\hline
\end{tabular}

Base: 88 respondentes dos Questionários 1 e 2

\subsection{COMPARANDO OS RESULTADOS}

A Tabela 5 resume os resultados obtidos a partir das quatro formas de análise. As conclusões que emergem a partir da análise dessa tabela são as seguintes:

$\checkmark$ Os atributos com os quais os respondentes manifestaram mais insatisfação foram "erros" e "segurança".

$\checkmark$ A forma de análise mais imprecisa é o modelo I/S. Por se basear numa análise gráfica, este não permite ordenar os atributos. É possível apenas distinguir aqueles que se localizam em cada um dos quadrantes e dar-lhes o tratamento gerencial recomendado.

$\checkmark$ A análise de gap e a abordagem multiplicativa têm resultados mais próximos entre si do que os demais modelos.

$\checkmark$ Considerando que os resultados obtidos para cada uma das análises é diferente, a segunda hipótese também deve ser rejeitada. 


\section{Tabela 5 - Comparação entre as Formas de Análise de Satisfação}

\begin{tabular}{l|l|l|l}
\hline \multicolumn{1}{c|}{ SATISFAÇÃO SIMPLES } & \multicolumn{1}{c|}{ ANÁLISE DE GAPS } & MODELO I/S & \multicolumn{1}{c}{ ABORDAGEM MULTIPLICATIVA } \\
\hline Erros & Erros & Erros & Erros \\
\hline Segurança & Segurança & Segurança & Segurança \\
\hline FAQ & Manter contato & & Manter contato \\
\hline Instruções & Navegabilidade & & Instruções \\
\hline Classificar amigos & Conhecer pessoas pelo perfil & & Navegabilidade \\
\hline Saber se estou conectado & Instruções & & Conhecer pessoas pelo perfil \\
\hline Conhecer pessoas pelo perfil & Participar de comunidades & & FAQ \\
\hline Navegabilidade & Cadastrar perfil & & Saber se estou conectado \\
\hline Contato & Saber se estou conectado & & Cadastrar perfil \\
\hline Cadastrar perfil & Visual & & Visual \\
\hline Visual & FAQ & & Classificar amigos \\
\hline Participar de comunidades & Classificar amigos & & Participar de comunidades \\
\hline
\end{tabular}

\section{DISCUSSÃO}

Os resultados do estudo empírico rejeitaram as duas hipóteses propostas e apresentaram diferenças que devem ser analisadas mais atentamente.

O questionário 2, em que a importância foi avaliada por meio de uma escala de diferencial semântico, foi o que teve mais atributos com notas que variavam desde o mínimo possível (1) até 0 máximo (10), ou seja, todos os atributos receberam notas mínimas e, com exceção do atributo "visual", todos também receberam notas máximas.

Em termos de variabilidade, o questionário 3, em que a importância foi obtida por meio de uma escala de soma constante, foi considerado o melhor. Com exceção do atributo "manter contato", todos os demais atributos receberam notas máximas que variavam entre 15 e 38; também, com exceção de "manter contato", todos os atributos receberam nota mínima 0.

Já no questionário 4, em que a importância foi obtida por meio de uma escala de ordenamento, as variações entre os atributos foram relativamente menores que nos outros questionários.

$O$ fato de as respostas variarem mais na escala de soma constante permite inferir que as importâncias ficam mais destacadas: o atributo "manter contato", por exemplo, teve nota máxima 90, enquanto 0 atributo "participar de comunidades" teve nota máxima 38. 0 atributo "manter contato" recebeu a nota mais alta entre todos os atributos (90) e teve a mais alta nota mínima (5). Em segundo lugar, está 0 atributo "participar de comunidades", com nota máxima 38. Já 0 atributo "classificar amigos" foi o que obteve a menor nota máxima: 15.

No caso da segunda hipótese, as quatro técnicas realmente trouxeram resultados diferentes, assim como ocorreu com o estudo empírico descrito por Fontenot et al. (2005). No entanto, as autoras utilizam uma maneira bastante simplificada de analisar os dados, apenas ordenando as médias, ou índices, para atribuir um nível de importância a cada um. É muito provável que, devido aos índices muito próximos, encontrados no estudo descrito no texto, não haja diferença significativa entre as médias dos atributos, por isso é desaconselhável classificá-los apenas pela ordenação de suas médias.

Além disso, na técnica da abordagem multiplicativa, a importância e a insatisfação com os atributos têm pesos iguais na elaboração do índice ponderado. Conceitualmente é difícil estabelecer qual das medidas deve ter maior peso. Essa é uma questão que fica para próximos estudos: qual 0 peso ideal a ser considerado para o índice de insatisfação e para a importância? 


\section{CONCLUSÃO}

Este trabalho demonstra que, apesar de as três escalas para se obter a importância declarada dos atributos e os quatro métodos de análise da satisfação gerarem resultados diferentes, há consistência entre os dados. Para efeito de conhecimento e para uma análise generalista, não há problemas em optar-se por uma forma em detrimento de outra. No entanto, as implicações surgem caso os dados sejam utilizados não para escolher atributos a serem melhorados, e sim para deslocar esforços de áreas que estão bem avaliadas para áreas que estão mal avaliadas. Nesse caso, é altamente recomendável analisar os dados de várias formas diferentes, reunindo, ainda, 0 conhecimento proveniente da empresa e de outras pesquisas, com o objetivo de comparar informações e analisá-las em conjunto profissionais experientes.

Os resultados obtidos por meio do levantamento de dados primários demonstram que as escalas não trazem resultados exatamente iguais, mas, assim como no estudo de Pilli (2004), há semelhanças entre os dados obtidos. Com relação às formas de análise, os resultados também diferem. Conforme foi apresentado, 0 modelo importância-satisfação apresentou resultados contraditórios se comparados aos obtidos por meio da análise de gap.

$\mathrm{E}$, embora os resultados condigam com os obtidos por pesquisas anteriores, ressalta-se que 0 presente estudo possui algumas limitações que podem ter dificultado a acuracidade dos resultados. A primeira limitação diz respeito ao número reduzido de respondentes em cada uma das amostras, o que aumenta a probabilidade de erros nos resultados. A segunda limitação refere-se à maneira conveniente como a amostra foi selecionada. A utilização do método de bola de neve não garante que a amostra seja representativa em relação aos demais usuários do Website.

Por outro lado, alguns pontos não ficaram completamente esclarecidos e podem ser aprofundados em estudos futuros, conforme enumerado a seguir:

Será que as diferenças entre as escalas se mantêm em outros estudos com temas (produtos e serviços) diferentes?

$\checkmark$ Considerando os resultados relativamente contraditórios da análise de gap e do modelo importância-satisfação, qual das formas mais se aproxima da realidade?

$\checkmark$ Quais são os "pesos ideais" a serem atribuídos para a importância e a satisfação com os atributos na análise de abordagem multiplicativa?

Com este estudo, os autores esperam ter contribuído para a discussão da pesquisa de satisfação, salientando que, em uma pesquisa de satisfação, tanto a importância dos atributos quanto a satisfação com os atributos podem variar, dependendo do tipo de escala utilizado e do método pelo qual se analisa os dados.

\section{REFERÊNCIAS}

Chavda, Dinyar. "Measuring Satisfaction Isn't Enough." Quirk's Marketing Research Review, v. 6, Outubro, 2002.

$\mathrm{CHU}, \mathrm{R}$. Stated importance versus derived importance of customer satisfaction measurement. Journal of Services Marketing, v.16, n. 4, p. 285-301, 2002 
FONTENOT, G.; HENKE, L.; CARSON, K. Take Action on Customer Satisfaction. Quality Progress, v. 38, n. 7, p. 40-47, 2005.

HANSON, R. Determining Attribute Importance. Quirks Marketing Research Review, v. 6, Outubro, 1992.

KIVETZ, R.; SIMONSON, I. The Effects of Incomplete Information on Consumer Choice. Journal of Marketing Research, v. 37, n. 4, p. 427-448, 2000.

MALHOTRA, Naresh K. Pesquisa de Marketing: uma Orientação Aplicada. São Paulo: Bookman, 2001.

YOO, B.; DONTHU, N. Developing a Scale To Measure the Perceived Quality of na Internet Shopping Site (SITEQUAL), Quarterly Journal of Electronic Commerce, v.2, n.1, p. 31-46, 2001

PILLI, Luis. Modelagem da importância dos atributos de produtos e serviços em estudos de satisfação, Trabalho apresentado no 1․ Congresso Brasileiro de Pesquisa, São Paulo, 2004.

\title{
CLIENT SATISFACTION RESEARCH
}

\begin{abstract}
Scales and analytical methods Abstract This study compares the use of three types of scaling methods (semantic differential, rank-order, and constant sum) and three types of data analysis methods (simple attribution of satisfaction, gap analysis, importance- satisfaction model, and multiplicative approach) for the measurement of services and products attributes satisfaction. The objective of the study is to discuss the use of different scales and data analysis tools by comparing the results provided by each of them. To test the hypotheses, data was gathered using an electronic, structured questionnaire and the snowball sampling method. The findings reveal that both scales and data analysis methods provided significantly different results. The conclusion is that scales and data analysis methods can influence the conclusions to be drawn from the same data set.
\end{abstract}

Key-words: Consumer satisfaction. Client service. Marketing research. 


\section{ANEXO I}

COMPARATIVO DAS MÉDIAS DE IMPORTÂNCIA DOS ATRIBUTOS - ESCALA DE DIFERENCIAL SEMÂNTICO

\begin{tabular}{|c|c|c|c|c|c|c|c|c|c|c|c|c|c|}
\hline & & $\begin{array}{l}\text { Manter } \\
\text { Contato }\end{array}$ & Segurança & Erros & Navegabilidade & $\begin{array}{l}\text { Participar de } \\
\text { Comunidades }\end{array}$ & $\begin{array}{l}\text { Conhecer } \\
\text { Pessoas } \\
\text { Pelo Perfil }\end{array}$ & $\begin{array}{c}\text { Cadastrar } \\
\text { Perfil }\end{array}$ & Visual & $\begin{array}{c}\text { Saber se } \\
\text { Estou } \\
\text { Conectado }\end{array}$ & Instruções & FAQ & $\begin{array}{l}\text { Classificar } \\
\text { Amigos }\end{array}$ \\
\hline & & 8,58 & 8,43 & 8,07 & 8,00 & 7,93 & 7,33 & 6,76 & 6,49 & 6,45 & 6,22 & 5,36 & 4,92 \\
\hline Manter Contato & 8,58 & & & & & & & & & & & & \\
\hline Segurança & 8,43 & n.s. & & & & & & & & & & & \\
\hline Erros & 8,07 & $* * *$ & $* * *$ & & & & & & & & & & \\
\hline Navegabilidade & 8,00 & $* * *$ & $* * *$ & n.s. & & & & & & & & & \\
\hline Participar de Comunidades & 7,93 & $* * *$ & $* * *$ & n.s. & n.s. & & & & & & & & \\
\hline Conhecer Pessoas Pelo Perfil & 7,33 & $* * *$ & $* * *$ & $* * *$ & $* * *$ & $* * *$ & & & & & & & \\
\hline Cadastrar Perfil & 6,76 & $* * *$ & $* * *$ & $* * *$ & $* * *$ & $* * *$ & $* * *$ & & & & & & \\
\hline Visual & 6,49 & $* * *$ & $* * *$ & $* * *$ & $* * *$ & $* * *$ & $* * *$ & n.s. & & & & & \\
\hline Saber se Estou Conectado & 6,45 & $* * *$ & $* * *$ & $* * * *$ & $* * *$ & $* * *$ & $* * *$ & n.s. & n.s. & & & & \\
\hline Instruções & 6,22 & *** & $* * *$ & $* * *$ & $* * *$ & $* * *$ & $* * *$ & n.s. & n.s. & n.s. & & & \\
\hline FAQ & 5,36 & $* * *$ & $* * *$ & $* * *$ & $* * *$ & $* * *$ & $* * *$ & $* * *$ & $* * *$ & $* * *$ & $* * * *$ & & \\
\hline Classificar Amigos & 4,92 & $* * *$ & $* * *$ & $* * *$ & $* * *$ & $* * *$ & $* * *$ & $* * *$ & $* * *$ & $* * *$ & $* * *$ & n.s. & \\
\hline
\end{tabular}

${ }^{* * *}$ Significa diferença estatística ao nível de confiança de 95\% ( $\left.p<0,05\right)$ 


\section{ANEXO II}

COMPARATIVO DAS MÉDIAS DE IMPORTÂNCIA DOS ATRIBUTOS - ESCALA DE SOMA CONSTANTE

\begin{tabular}{|c|c|c|c|c|c|c|c|c|c|c|c|c|c|}
\hline & & $\begin{array}{l}\text { Manter } \\
\text { Contato }\end{array}$ & $\begin{array}{l}\text { Participar de } \\
\text { Comunidades }\end{array}$ & $\begin{array}{c}\text { Conhecer } \\
\text { Pessoas Pelo } \\
\text { Perfil }\end{array}$ & Segurança & Navegabilidade & $\begin{array}{c}\text { Cadastrar } \\
\text { Perfil }\end{array}$ & $\begin{array}{l}\text { Saber se } \\
\text { Estou } \\
\text { Conectado }\end{array}$ & Erros & Visual & Instruções & FAQ & $\begin{array}{c}\text { Classificar } \\
\text { Amigos }\end{array}$ \\
\hline & & 20,93 & 10,94 & 8,69 & 8,54 & 8,16 & 8,13 & 7,00 & 6,68 & 5,88 & 5,53 & 5,02 & 4,48 \\
\hline Manter Contato & 20,93 & & & & & & & & & & & & \\
\hline Participar de Comunidades & 10,94 & **** & & & & & & & & & & & \\
\hline Conhecer Pessoas Pelo Perfil & 8,69 & $* * *$ & $* * *$ & & & & & & & & & & \\
\hline Segurança & 8,54 & $* * *$ & $* * *$ & n.s. & & & & & & & & & \\
\hline Navegabilidade & 8,16 & **** & $* * *$ & n.s. & n.s. & & & & & & & & \\
\hline Cadastrar Perfil & 8,13 & **** & **** & n.s. & n.s. & n.s. & & & & & & & \\
\hline Saber se Estou Conectado & 7,00 & $* * *$ & $* * *$ & n.s. & n.s. & n.s. & n.s. & & & & & & \\
\hline Erros & 6,68 & **** & $* * *$ & **** & $* * *$ & n.s. & n.s & n.s. & & & & & \\
\hline Visual & 5,88 & **** & $* * *$ & **** & $* * *$ & $* * *$ & $* * *$ & n.s. & n.s. & & & & \\
\hline Instruções & 5,53 & $* * *$ & $* * *$ & $* * *$ & $* * *$ & $* * *$ & $* * *$ & $* * *$ & n.s. & n.s. & & & \\
\hline FAQ & 5,02 & **** & **** & **** & $* * *$ & $* * *$ & **** & $* * *$ & n.s. & n.s. & n.s. & & \\
\hline Classificar Amigos & 4,48 & $* * *$ & $* * *$ & $* * *$ & $* * *$ & $* * *$ & $* * *$ & $* * *$ & $* * *$ & $* * *$ & n.s. & n.s. & \\
\hline
\end{tabular}

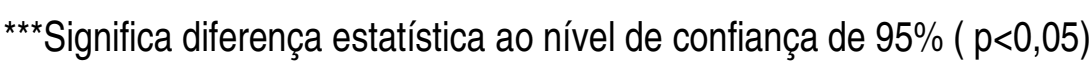




\section{ANEXO III}

\section{COMPARATIVO DAS MÉDIAS DE IMPORTÂNCIA DOS ATRIBUTOS - ESCALA DE ORDENAMENTO}

\begin{tabular}{|c|c|c|c|c|c|c|c|c|c|c|c|c|c|}
\hline & & $\begin{array}{l}\text { Manter } \\
\text { Contato }\end{array}$ & Segurança & Navegabilidade & $\begin{array}{l}\text { Participar de } \\
\text { Comunidades }\end{array}$ & $\begin{array}{l}\text { Cadastrar } \\
\text { Perfil }\end{array}$ & $\begin{array}{c}\text { Pessoas Pelo } \\
\text { Perfil }\end{array}$ & $\begin{array}{c}\text { Estou } \\
\text { Conectado }\end{array}$ & Erros & Instruções & Visual & FAQ & $\begin{array}{c}\text { Classificar } \\
\text { Amigos }\end{array}$ \\
\hline & & 2,69 & 4,44 & 5,10 & 5,41 & 6,23 & 6,43 & 6,85 & 7,05 & 7,69 & 8,32 & 8,54 & 9,25 \\
\hline Manter Contato & 2,69 & & & & & & & & & & & & \\
\hline Segurança & 4,44 & **** & & & & & & & & & & & \\
\hline Navegabilidade & 5,10 & *** & n.s. & & & & & & & & & & \\
\hline Participar de Comunidades & 5,41 & *** & n.s. & $* * *$ & & & & & & & & & \\
\hline Cadastrar Perfil & 6,23 & $* * *$ & $* * *$ & $* * *$ & $* * *$ & & & & & & & & \\
\hline Conhecer Pessoas Pelo Perfil & 6,43 & $* * *$ & $* * *$ & $* * *$ & $* * *$ & n.s. & & & & & & & \\
\hline Saber se Estou Conectado & 6,85 & $* * *$ & $* * *$ & $* * *$ & $* * *$ & n.s. & n.s. & & & & & & \\
\hline Erros & 7,05 & **** & $* * *$ & *** & $* * *$ & n.s. & n.s. & n.s. & & & & & \\
\hline Instruções & 7,69 & $* * *$ & $* * *$ & *** & *** & *** & **** & n.s. & n.s. & & & & \\
\hline Visual & 8,32 & *** & $* * *$ & $* * *$ & $* * *$ & $* * *$ & **** & $* * *$ & $* * *$ & n.s. & & & \\
\hline FAQ & 8,54 & **** & **** & *** & *** & **** & **** & *** & $* * *$ & $* * *$ & n.s & & \\
\hline Classificar Amigos & 9,25 & $* * *$ & $* * *$ & **** & $* * *$ & $* * *$ & $* * *$ & $* * *$ & $* * *$ & $* * *$ & $* * *$ & n.s. & \\
\hline
\end{tabular}

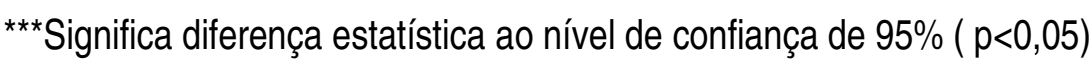

